\title{
ZUR GESCHICHTE \\ DES ENGLISCHEN GERUNDIUMS.
}

An der spitze des dritten heftes des 45. bandes der Englischen Studien veröffentlicht George 0 . Curme einen beachtenswerten aufsatz, dessen gegenstand der ist: den weg aufzuzeigen, auf welchem das englische verbalsubstantiv die gerundialen geltungen und verwendungen erreichte, welche es heute noch auszeichnen. Verstehe ich Curme recht, so argumentiert er so: da auch andere germanische sprachen das bedürfnis nach einem gerundium empfanden, so ist es nur natürlich, dals auch die englische sprache dieses bedürfnis empfand. In jenen lassen sich die beweise für die befriedigung dieses bedürfnisses gegen ende des zwölften jahrhunderts nachweisen; in der englischen sprache wesentlich früher und zwar zu einer zeit, wo an sich schon der einflufs einer fremden sprache völlig ausgeschlossen ist, abgesehen vom Lateinischen, dessen einflufs hier nicht wesentlich in frage kommt.

Die betrachtung der einzelnen entwicklungsstufen des englischen gerundiums einer späteren seite vorbehaltend, wollen wir hier zunächst stehen bleiben, um die obigen 'voraussetzungen' Curme's genauer zu betrachten.

Für das bestreben der kontinentalen germanischen sprachen, gerundiale formen zu entwickeln, führt Curme (p. 360) den beleg an: dâ wart vil michel grüezen die lieben geste getân Nib. L.; ich kenne noch mehr: Paul in seiner MHD. Gramm. $\S 296$ bietet noch vier oder fünf weitere, und Stoett in seiner MNL. Spraakkunst $\S 278$ bietet gleichfalls eine reihe von derartigen konstruktionen: Een overliden alle ghescapenheit; Toten stortene onse bloet; etc. etc. Und ähnliches oder gleiches bietet 
auch das ME.: Jurh herborzin wrecche men V. \& V., ein beleg, den Curme selbst, allerdings in unrichtiger verbindung ${ }^{1}$ ) bringt. Aber, meine ich, die schwierigkeit der frage liegt doch weniger darin, dals mehrere der germanischen schwestersprachen diesen drang nach gerundialen formen empfanden und bewiesen, sie liegt darin, dals allein das Englische diesen am verbalsubstantive zur geltung brachte.

Die erstere schwierigkeit zu lösen ist schon nicht leicht, denn wie kam es, müssen wir uns fragen, dafs gerade die drei genannten germanischen sprachen diesen drang empfanden und nicht die anderen; noch viel schwerer zu lösen ist aber die letztere: wie kam es, dafs gerade und allein das Englische bei der umschau nach geeigneten formen auf das verbalsubstantiv verfiel, noch dazu, nachdem es eine zu gerundialem ausdruck passende form schon im infinitiv gefunden hatte. Curme wird doch nicht behaupten wollen, dafs jenes den verbalen gehalt energischer zum ausdruck brachte als dieses. Und er wird doch, andererseits, nicht behaupten wollen, dals das englische verbalsubstantiv jenen gehalt energischer zum ausdruck brachte als das der anderen genannten schwestersprachen. Gegenwärtig freilich besitzt das Deutsche ein gerundartiges verbalsubstantiv. Curme belegt es aus den Hamburger Nachrichten vom 20. Nov. 1904, und es würde ihm leicht sein, melır belege zu finden. Wir besitzen es aber nur als eine nachahmung des englischen und auch nur, seit englisches wesen und englische kultur ein so hohes ansehen bei uns geniefst. Es würde Curme schwer werden, derartige konstruktionen wie "eine befassung der semstwos mit der frage einer russischen verfassung" vor, sagen wir, der mitte des 19. jahrhunderts nachzuweisen.

Mit dem hinweise auf die germanischen schwestersprachen ist also nichts gewonnen. Er erklärt nicht nur nichts, er läfst im gegenteil die verhältnisse im Englischen nur noch rätselhafter erscheinen.

Und nun zur frage des beginnes der gerundialen bewegung. Es ist oben gesagt, dals diese nach Curme's ansicht im Englischen um etwa ein viertel jahrtausend früher einsetzt als in

1) Dieser wie die aus den anderen sprachen sind substantivierte infinitive mit verbaler rektion, die übrigen mit to sind 'verbale' infinitive. 
den anderen germanischen sprachen: also in der besten altenglischen zeit. Sehen wir nun die belege, die er hierfür als beweise beibringt, uns etwas genauer an.

Zum beweise, dals das ae. verbalsubstantiv gerundiale geltung $\mathrm{zu}$ entwickeln beginnt, führt Curme (p. 351) einen Bedabeleg an, in welchem nach seiner ansicht ein verbalsubstantiv durch einen adverbialen ausdruck näher bestimmt ist, nämlich: pret ... ge pa pegnunge fulwihte ... gefyllen. Hätte er jedoch das lateinische original beachtet ut ministerium baptizandi .... conpleatis und aufserdem in dem ae. texte, in dessen $\mathrm{B}$. I noch eine ganze anzahl derartiger konstruktionen in noch viel deutlicherer fassung vorkommen, etwas weiter gelesen, so würde er schon selbst bemerkt haben, dafs mit diesem belege nichts anzufangen ist. Curme hat eben nicht beachtet, dais fullwiht, obzwar es im jüngeren AE. regelmälsig als neutrum verwendet wird, doch ursprünglich ein femininum (Sievers, Gramm. § 267 b) war, und dals es die Beda-handschriften $\mathrm{O}$ und $\mathrm{Ca}$ ausschliefslich noch als solches kennen und gebrauchen. Aelfreds übersetzung ist also wörtlich genau, und fulwihte ist der genitiv.

Ist nun überhaupt dadurch, dals eine adverbielle bestimmung in nähere verbindung zu einem verbalsubstantive tritt, schon dessen gerundartige natur bewiesen? Durchaus nicht, denn diese verbindung findet sich bei substantiven beliebiger bildung. Ich erinnere an die von Wülfing angeführten hider cyme, heonan sid, die mit fraglicher berechtigung manchmal zusammengeschrieben werden, weiterhin an die wohl stets getrennten noenig after yldo; min iu magister; mid ofercyme semninga deapes, von Wülfing sämtlich Beda entnommen, und hierher gehört nun auch der von Curme (p. 353) angeführte beleg [seo] on morgen deagung aus crepusculum ... matutinum; auch die von mir bei weiterer lektüre gefundenen Angelcynnes cyme on Breotene ib. I 16 aus adventus eorum in Britanniam und gehet ... toweard rice butan ende ib. 25 aus regnum sine fine ... futurum gehören schliefslich hierher.

Es war also für das Altenglische durchaus nichts ungewöhnliches, substantiva unmittelbar mit adverbiellen bestimmungen zu verbinden, ebensowenig wie es dies für das 
heutige Englisch oder für das heutige Deutsche ${ }^{1}$ ) ist. Und da das verbalsubstantiv ein substantiv ist wie jedes andere, so ist es irrig, aus seiner verbindung mit einem adverbium $z u$ folgern, dafs es damit und dadurch zu einer anderen wortkategorie übergetreten sei.

So wenig charakteristisch für das gerundium seine verbindung mit einem adverb ist, so unzweideutig kennzeichnend für dasselbe ist seine verbindung mit einem akkusativ-objekte. Die mühe, welche Curme aufwendet, dieses kennzeichen noch für das ae. verbalsubstantiv nachzuweisen, ist darum wohl erklärlich. Dürfte ich einen vergleich aus dem rennsport heranziehen, so würde ich sagen, diese verbindung ist nicht nur das erste, sie ist das einzige bedeutende hindernis auf der entwicklungsbahn des verbalsubstantivs zum unzweideutigen gerundium: ist dieses überwunden, so ist der rest wenig mehr als ein 'walk-over'.

Es ist nun auf den ersten blick ansprechend, wenn Curme (p. 355) behauptet, daIs die zusammensetzungen, die aus objekt und einem beliebigen substantive verbalen gehaltes bestehen, wie ciricsocn, mannsielen, sopsegen, landbegenga, blodspiwung etc. dem grammatisch ungebildeten leicht als aus akkusativobjekt und regens bestehend erscheinen konnten, und da sie dies leicht konnten, auch $\mathrm{zu}$ nachweisbaren verwechselungen führen mufsten. Dagegen ist jedoch zu bemerken, dafs dann nicht einzusehen wäre, warum solche verwechselungen gerade auf die verbalsubstantive auf -ung sich hätten beschränken sollen, dafs aber in wahrheit weder für die verbalsubstantive auf -ung, noch für irgend welche anderen verbalsubstantive sich aus gut altenglischer zeit beweise beibringen lassen, dafs eine derartige verwechselung je stattgefunden hätte. Im gegenteil, jene komposita bleiben unter allen umständen komposita und wenn hier eine auflösung statthaben sollte, so kann an stelle des das objekt darstellenden ersten substantives

1) Es ist deshalb auch nicht zu verstehen, wie Curme behaupten kann (p. 355), dafs dem englischen the ride home, the ride back etc. das deutsche der heimritt, der rïckweg etc. genau (closely!) entspricht, und dals die ' components of a German compound substantive are never seperated'. Neben diesen freilich gut deutschen heimritt etc. stehen bekanntlich die ebenso gut deutschen 'der ritt nach hause', 'der weg zurück', 'die fahrt hinunter' etc. etc. 
nur der objektive genitiv, nie aber der objektive akkusativ, eintreten; dafür ist ein guter beweis der von Curme (p. 352) angeführte Lindisf.-beleg: non cessavit osculari pedes meos = ne blann cossetunges foeta mine, dessen schreiber zunächst pedes meos ganz wörtlich mit foet mine übersetzte, dann aber in rücksicht auf das von ihm gewählte verbal-substantiv dem ersteren das - $a$ des (objekt-) genitivs anfügte, ohne jedoch aus dem attribut. mine ein min oder minra zu machen. Dals dies und das oe von foeta zu der verbesserten fassung nicht stimmte, scheint ihn, wie in anderen fällen, nicht gestört zu haben. Nach Sievers (Gramm. $§ 281$ a. 2) freilich soll das einmalige (!) foeta ein wohlgemeinter genitiv sein; dies ist aber schon im hinblick auf mine unwahrscheinlich. Die beiden bildungen bleiben eben im guten Altenglischen so scharf getrennt, und so fest gefügt war die struktur der alten sprache, dals hier eine vermischung nie eintritt und dals man in einer bindung, deren erster teil aus einem endungslosen substantive (dem stamme) besteht, unweigerlich genötigt ist, eines jener uralten komposita zu sehen, die wir an der spitze dieses paragraphen anführten. Auch bei bildungen, die eine viel stärkere verbale kraft besitzen als das verbalsubstantiv auf -ung, steht das objekt im genitiv: pone forhycgend ura goda Beda, done godes andettere ib. und deshalb ist auch blodspiwung und bocraeding nie als 'spitting blood' und 'reading books' zu fassen, wenn es Curme (p. 355) auch so übersetzt, sondern als bloodspitting und bookreading. Und darum war es auch überflüssig, in der form becraedinge die möglichkeit der oben geschilderten verwechselung zu erkennen (p. 356), bewies doch schon die variante boca radinge (vgl. Bosw.-Toller s. v.), dals der Altengländer die form bec richtig als genitiv verstand. In der art, wie Curme die möglichkeit einer solcher verwechselung beweist, ist er besonders unglücklich: den beleg Jonne heo heora becradon beeodon begleitet er mit den worten: 'The writer has never met a genitive in an infinitive compound either in English or German.' Gewils, ich auch nicht! Aber -radon ist eben gar kein infinitiv, sondern der akkusativ des abstraktums roede = 'lectio, reading, lesson' und darum das voranstehende bec eben wieder der objektive genitiv. $\mathrm{Zu}$ der äufserung, dals "the first component is not a bare stem but an inflected accusative plural and the first step toward the 
final dissolution of the compound had been taken", lag also nicht der geringste anlals vor. Sie beruht auf eben derselben unbedachten vorausnahme, die wir schon oben zu rügen hatten.

Hiermit sind wir am ende mit den beweisen, welche nach Curme's ansicht dartun sollen, dafs der trieb, gerundiale formen $\mathrm{zu}$ entwickeln, schon in altenglischer zeit sich $\mathrm{zu}$ äufsern beginnt; und da wir hier von diesem triebe nichts zu spüren im stande waren, bleibt, uns nichts übrig, als unser augenmerk zu richten auf die folgende oder besser (da Curme's zeitlich folgende belege dem ende des 12. jahrh. entstammen) die übernächste: die frühmittelenglische zeit. Hiermit treten wir aber in eine sprachperiode ein, in der jener bewufste fremdsprachliche einfluis, den Curme unter allen umständen zu eliminieren gedachte, sehr wohl wirksam werden konnte. Curme behauptet zwar bei gelegenheit (p. 377): "This clumsy construction .... is found in the twelfth century before French had in any way influenced English syntax." Die nicht wenigen romanischen parallelen $\mathrm{zu}$ ausschliefslich frühmittelenglischen konstruktionen, die der verfasser in meinen späteren untersuchungen finden konnte, hätten ihn aber wohl eines anderen und besseren belehren können.

$\mathrm{Zu}$ der frage des romanischen einflusses auf die englische syntax im allgemeinen sage ich hier nur folgendes:

Da es (begreiflicher weise) nicht nur mir, sondern jedem ernsten gelehrten daran liegen mufs, dafs der gegenwärtige stand der nun schon recht alten kontroverse nicht verschoben wird, so halte ich es für meine pflicht, gegenüber Curme's ansicht "This remark (scil. of Kellner's: 'this construction is due to the French original!') belongs to a long list that ought to be stricken out of our learned literature; they will soon appear queer to readers for we are fast coming to the clear insight that French influence upon English syntax has been greatly overestimated" (p. 377), die bisherigen ergebnisse jener kontroverse hier kurz in erinnerung zu bringen: Da vernunftgemäls und erfahrungsmälsig jeder stärkere lexikalische einflufs ohne syntaktischen einflufs weder denkbar noch in der wirklichkeit nachweisbar ist, so hat, da der gewaltige lexikalische einflufs des Romanischen auf das Englische nicht geleugnet werden kann, derjenige, welcher den entsprechenden 
einflufs auf syntaktischem gebiete bestreitet, die pflicht, jeden einzelnen fall, jede einzelne romanischenglische parallele dadurch zu entkräften, dafs er eine altenglische parallele, oder wenigstens eine solche, aus den nächstverwandten germanischen sprachen beibringt. Bezüglich der letzteren sind aber, wie ich ausdrücklich erwähne, im allgemeinen nur belege aus der ältesten sprachperiode (abgesehen vom Altnordischen) beweiskräftig, weil die jüngeren perioden unter demselben, wenn auch schwächeren, einflusse standen wie das Mittelenglische. Für das Mittelniederländische lälst sich dies leicht beweisen. Schwerer ist der beweis für das Mittelhochdentsche zu erbringen, da hier für diese frage, abgesehen von anfängen und versuchen, wie in den wörterbüchern von $\mathrm{H}$. Paul und Weigand (Bahder-Hirt-Kant), noch das meiste zu tun ist.

Ergänzend füge ich folgendes bei:

Schon in der einleitung zu meinem Indefinitum, Anglia 26 p. $470 \mathrm{ff}$,, wies ich auf grund der damaligen ethnologischen verhältnisse darauf hin, dals in weiten schichten des englischen volkes im 13. und 14. jahrh. eine weitgehende sprachmischung bestanden haben müsse, und ich habe an eben der stelle einige literarische belege beigebracht, die für diese sprachmischung den augenscheinlichen beweis liefern.

Ich bin nun heute in der lage, einen weiteren beweis für die existenz dieser mischsprache beibringen zu können. Nach Godefroy s. v. patroullart schreibt das MS. Soiss. 210 der Somme des V. et V.: Il prie Deu patroullart comme cil qui prie Den moitié franceis moitié latin und diese fassung wird auf den verfasser Laurent selbst zurückgehen, dem als Franzosen diese art misch-masch (patroullart von patrouil = 'puddle', 'mud' O. D.) naturgemäfs am nächsten lag. Diese originalfassung aber wird von dem schreiber des MS. Maz. 809 abgeändert wie folgt: il parole a Dieu patroulart comme cil qui parole moitié françois moitié anglois! Und es ist klar, dals erstens - dieser schreiber, wenn nicht selbst ein Normanne, so doch mit den sprachverhältnissen der Normannen in England engvertraut war, zweitens, dafs er hier nicht auf ein mit gelegentlich englischen worten gespicktes Normannisch, sondern auf ein etwa zu gleichen teilen aus Englisch und Normannisch zusammengesetztes misch-masch sich bezieht; dies wäre dann 
also eine theoretische bestätigung der existenz jener mischsprache, wie ich sie in der einleitung zu meinem "Indefinitum" (p. 471) aus Wright's Pol. Songs so glücklich belegen konnte, und ich will hier nochmals hervorheben (vgl. Indef., Einl. p. $466 \mathrm{ff}$.), dals gerade die hier gemeinte mischsprache, die sprache der Englisch radebrechenden Normannen von eminenter bedeutung für die weiterentwickelung der englischen sprache gewesen ist. Zum schlusse bemerke ich: da Laurent die Somme 1279 schrieb und wir die abschrift des MS. Maz. kaum vor ende der 90er jahre ansetzen können, der sonst so selbständige bearbeiter Dan Michel aber an der bewuIsten stelle gehaltlich nichts zu ändern findet (he spekp to god patroyllart, ase pe ille pet spekp half englis and half urenss), so erhalten wir für die existenz jener mischsprache die zeit von ca. 1290 bis (wenigstens) 1340, dem jahre der vollendung der Ayenbite. Von dem terminus a quo, dem jahre, in welchem jene mischsprache aufzufallen begann, werden wir weiterhin noch zu reden haben.

Ob nun aber eine solche hochgradige sprachmischung ohne gleichzeitige syntaktische kreuzungen sich denken lälst, das zu erwägen, kann ich getrost dem leser überlassen.

Hiermit lassen wir diesen gegenstand fallen. Es wird sich später mehrfach gelegenheit flnden, das hier gesagte weiter auszuführen und materiell zu belegen.

Und nun zu dem gange der entwicklung, den das neue gerundium in der folgezeit genommen. Nach Curme entwickelt das gerundium alle seine heutigen gebrauchsweisen aus seiner eigenen kraft, aus sich selbst, allein. Weder der infinitiv noch das part. präsentis treten ihm hilfreich zur seite. Curme gibt nur zu, dafs im laufe der zeit gelegentlich das eine das andere verdrängt habe, das eine dem anderen gewichen sei. Dafs das eine an dem aufbau des anderen tätigen anteil genommen, und dals so namentlich auch das gerundium von der beihilfe eines der anderen oder beider nutzen gezogen, lälst er durchaus nicht gelten, und er läfst sich in dieser seiner überzeugung weder von Sweets ansicht (p. 372) noch von der der gelehrten des $0 . D$. (p. 369), noch von der anderer 'eminent scholars' beirren, von der meinigen ganz zu schweigen.

Dabei begegnen ihm eine reihe von versehen und verwechselungen. Infinitive erscheinen ihm als gerundien und 
umgekehrt, gerundien als partizipien und vice versa, und nicht nur in fällen, wo die schwierigkeit des stoffes den fehlgriff wohl erklärlich macht, sondern leider auch dort, wo eine strengere beachtung der laut- und formengeschichte den verfasser vorr einem irrtume hätte bewahren können. Es wäre kleinlich und aufserdem zeitvergeudung, diese fälle hier aufzuzählen. Der fachmann wird aus den im vorhergehenden besprochenen teilen ohnehin auf die folgenden nicht besprochenen zu schliefsen im stande sein. Und überdies wird aus dem, was ich weiterhin zu sagen haben werde, das meiste von dem, was hier hätte angemerkt werden können, sich von selbst korrigieren.

"The gerund can only be completely understood when its historical development has been carefully studied" sagt Curme auf p. 359, und ich möchte nicht geradezu behaupten, dals er diese seine ansicht in seiner arbeit nicht beherzigt hätte. Sein hauptfehler aber scheint mir der zu sein, dals er diese historische entwickelung gar zu sehr mit den augen des modernen Engländers durchforscht hat. Auf der höhe der entwickelung stehend, verfällt er, und nicht nur er, sondern der englisch-geborene überhaupt gar zu leicht in den fehler, moderne anschauungen und gedanken einer ausdrucksweise unterzulegen, deren entwickelung um drei, vier, fünf stufen hinter der ihm geläufigen zurückliegt. In den allermeisten fällen hatte jener ausdruck 'damals' einen ganz anderen gefühls- und gedankeninhalt, als er jetzt besitzt, und um sich seines voll und ganz zu bemächtigen, hat der moderne Engländer eine mühe und ausdauer aufzuwenden, die der der erlernung einer ihm völlig fremden sprache gleichkommt. Eine mühe, die er sich nur dadurch in etwas erleichtern könnte, dafs er sich eine der hinter der seinigen in der entwickelung zurückgebliebenen aber noch lebenden nächstverwandten sprachen, wie das Holländische oder Deutsche es ist, zu eigen macht, aber zu eigen macht in des wortes vollster bedeutung.

Und hiermit gerade berühren wir den punkt, von dem aus die holländische und deutsche (und weiterhin skandinavische) mitarbeit an themen der englischen sprachhistorie nicht blofs als duldbar, sondern geradezu als notwendig erwiesen werden kann: da unsere sprachen ihrer entwickelung nach dem Mittelenglischen des 13. oder 14. jahrhunderts entsprechen und mit 
diesem, ihren vorstufen, ihren entwicklungstendenzen nach, ihrem ganzen gefühls- und anschauungsgehalt nach, übereinstimmen, und da überdies kein Engländer unsere sprachen so meistern wird wie wir selbst, so sind wir die gegebenen, die geborenen mitforscher an der englischen sprachgeschichte, so weit diese sich mit syntaktischen fragen befalst.

Freilich werden wir unserseits, in den gegensätzlichen fehler verfallend, geneigt sein, in der lebenden sprache noch ältere zustände zu erblicken, die diese längst überwunden hat. Aber den folgen dieses fehlers werden wir Kontinentalen leichter entgehen können als der Engländer dem ihm drohenden, da unsere begabung für die erlernung fremder sprachen die des Engländers bei weitem übertrifft.

Die kontinentalen fachkollegen werden es mir verzeihen, wenn ich mich hier über dinge verbreite, die ihnen als selbstverständlichkeiten erscheinen müssen. Nach den hier und da von Engländern und Amerikanern geäufserten ansichten, ansichten, die sich auch in der im vorliegenden besprochenen arbeit Curmes nicht undeutlich offenbaren, sind diese dinge aber für jene durchaus keine selbstverständlichkeiten, und es ist deshalb vielleicht nicht unnütz, sie einmal offen und ungescheut auszusprechen.

Diese worte haben, wie ich es offen betone, nicht den zweck, die arbeiten der Kontinentalen im allgemeinen, oder im vorliegenden konkreten falle meine demnächst folgende eigene arbeit, auf ein überragendes piedestal zu stellen. Sie haben nur den zweck, eine gewisse anderwärts herrschende selbstüberschätzung zu bekämpfen und unsere stellung als die gleichberechtigter mitforscher $\mathrm{zu}$ begründen. Aus voller absicht habe ich deshalb oben den ausdruck 'mitforscher' gewählt. Denn da auf die geschilderte art unser aller fähigkeiten sich in der wünschenswertesten weise gegenseitig ergänzen, so haben wir keinen anlals 'to stryve as dide the houndes for the boon', sondern im gegenteil allen anlals uns zu vertragen, da nur so unsere wissenschaft $\mathrm{zu}$ dem rechte kommen kann, das ihr gebührt.

Meine im folgenden hefte zu veröffentlichende arbeit mag, wenn auch in anderer weise, ebenso $\mathrm{zu}$ tadel anlafs geben wie die Curmes. Sie ist überhaupt keine konkurrenzarbeit zu dieser, da sie vor mehr als 15 jahren geplant wurde als eine 
studie über den weg, auf welchem das Englische sein angestammtes partizipium präsentis verlor und auf welche weise es sein neues modernes entwickelte.

Seit mehr als 15 jahren, wie gesagt, habe ich belege für diese studie gesammelt, und ich hätte sie vielleicht schon veröffentlicht, wenn mir in bezug auf gewisse, glücklicherweise für unsere $z$ wecke untergeordnete, punkte das belegmaterial nicht zu unvollständig geschienen hätte.

Jetzt, da meine arbeit nach vielen richtungen hin vermehrt und erweitert worden ist, und da die Englischen Studien dieser an sich schon so interessanten frage einen so hervorragenden platz eingeräumt haben, habe ich kein bedenken mehr, eine arbeit $\mathrm{zu}$ veröffentlichen, die, wenn stellenweise auch jetzt noch ergänzungsbedürftig, ihrem plane nach mit der an jener stelle erschienenen, $\mathrm{zu}$ einem grofsen teile sich deckt.

Halle im Mai $1913 . \quad$ Eugen Ennenkel.

\section{Corrigendum.}

On some uses of the Indefinite Relatives in Old English and on the Origin of the Definite Relatives.

S. $292 \S 10$, a. Hogodon georne etc. and the note are to be left out.

Olaf Johnsen. 